\title{
Formation of benign tumours by stem cell misregulation
}

\author{
Matthieu Valet \& Patrick Narbonne*
}

Département de biologie médicale, Université du Québec à Trois-Rivières, Trois-Rivières, Québec, Canada G8Z 4M3.

*, Correspondence should be addressed to: patrick.narbonne@uqtr.ca

\begin{abstract}
Our tissues usually have just the right number of cells to optimally fulfil their function. Not enough cells within a tissue can lead to dysfunction, while too many cells result in a tumour. Yet, how this homeostatic balance is maintained remains poorly defined. Most differentiated cells within tissues have a finite lifespan and need to be replaced at a corresponding pace to maintain tissue homeostasis. These new differentiated cells are generated by proliferation of the stem/progenitor cells that serve the tissue. Work in simple invertebrates clearly suggests stem cells respond to at least two types of signals: niche signaling and growth factors. Niche signals promote the undifferentiated state by preventing differentiation, and thus allow for stem cell self-renewal. Growth factor sources comprise a systemic input reflecting the animal's nutritional status, and a localized, homeostatic feedback from the tissue that the stem cells serve. That homeostatic signal couples stem cell proliferation rates to the tissue's need for new differentiated cells. Evidence from simple organisms suggests two types of benign tumours can arise from deregulation of either niche or homeostatic signaling. Namely, constitutive niche signaling promotes the formation of undifferentiated "stem cell" tumours, while defective homeostatic signaling leads to the formation of differentiated tumours. We propose that these principles may be conserved and underlie benign tumour formation in humans, while benign tumours can evolve into cancer.
\end{abstract}


A benign tumour is an abnormal mass of extra cells within a tissue that lack the ability to invade neighboring tissues or metastasize. Hence, benign tumours are not cancerous and, depending on where they arise, are usually not an immediate life-threatening problem. However, benign tumours have the potential to become malignant ${ }^{1}$ and, as such, their formation may be considered a key early step in carcinogenesis. Most cells within early-stage benign tumours are typically welldifferentiated, albeit in rarer cases, they can be undifferentiated ${ }^{2,3}$. On the basis of recent advances in our understanding of stem cell regulation in simple invertebrates, we propose that benign tumours, undifferentiated and differentiated, each develop from a different type of stem cell misregulation. Namely, constitutive niche signaling causes the formation of undifferentiated "stem cell" tumours, while defective homeostatic signaling leads to the formation of differentiated tumours.

Keywords: stem cells; proliferation; tumour; benign tumour; cancer

\section{Stem cell regulation}

Stem cells (SCs) have a unique dual potential, showing both the capacities to perpetually selfrenew, and to differentiate into other cell types. Since the original demonstration of these capacities in hematopoietic $\mathrm{SCs}^{4}$, several other SC types have been discovered and characterized, such as the mammalian pluripotent embryonic stem cells (ESCs) ${ }^{5}$, and various adult SCs (also known as tissue-specific, tissue-resident, or multipotent SCs). Our understanding of in vivo SC regulation was however hindered by their limited accessibility in most systems, requiring complex surgical dissections and/or transplantation ${ }^{6}$. As such, the landmark introduction of in vitro SC culture revolutionized the accessibility of $\mathrm{SC}$ research by facilitating expansion, manipulation and microscopic observation of SCs ${ }^{5}$. Yet, $\mathrm{SCs}$ respond to various signals within a living organism and identifying signals responsible for SC maintenance and function quickly became an important question. Indeed, the existence of a specialized microenvironment regulating hematopoietic SC function was hypothesized ${ }^{7}$ but could not be readily demonstrated in this complex system. A range of SCs were thus discovered and characterized in simple invertebrate models that allow the effective combination of genetic, cell biological and molecular approaches, such as Drosophila ${ }^{8,9}$ and $C$. elegans ${ }^{10}$. This allowed for the discovery of the SC niche, niche signaling, and more recently, of additional mechanisms regulating SC proliferation rates. In the next two sections, we primarily use the $C$. elegans germline stem cells (GSCs) to illustrate how SCs are regulated in vivo, while these basic principles appear highly conserved in other organisms and SC types ${ }^{11-14}$.

\section{Regulation of stem cell fate by niche signaling}

The GSCs of C. elegans exist as two equivalent and homogeneous populations located at the distal extremities of each of the adult animal's two gonad arms (Fig. 1A-B). At each tip lies a somatic cell called the distal tip cell (DTC), which acts as the GSC niche ${ }^{10}$. GSCs divide symmetrically, giving rise to two daughters with equivalent fate potential ${ }^{11,15}$. However, the ongoing proliferation within the finite space of the distal gonad stochastically displaces GSC daughters away from the niche. GSC daughters therefore display a population asymmetry and progress towards differentiation as they move proximally, away from the DTC, within the gonadal tube ${ }^{11}$. The GSCs along with their proliferating progeny that have launched the differentiation program, but not yet begun overt differentiation, span a region termed the Progenitor Zone (PZ) (Fig. 1C).

The membrane-bound Delta-like ligand LAG-2, expressed by the DTC, was identified as a niche signal that prevents GSC differentiation by activating the Notch receptor GLP-1 at the 
GSC surface ${ }^{16,17}$. When either lag-2 or $g l p-1$ is mutated, or when the DTC is ablated, all GSCs are lost to differentiation, their default fate ${ }^{10,16,17}$. From thereon, niche signaling has been regarded as the signal(s) that instructs SC fate, preventing SC differentiation. When GLP-1 is activated, its intracellular domain is cleaved, translocates to the nucleus, and serves as a co-activator for the transcription factor LAG-1 (homologous to human C promoter Binding Factor, CBF1), which binds to and activates the transcription of two main target genes, $l s t-1$ and $s y g l-1^{18-21}$. LST-1 and SYGL-1, two Caenorhabditis-specific proteins, then combine with RNA-binding proteins FBF-1 and FBF-2 to repress three largely parallel, but partly redundant pathways that together promote differentiation (Fig. 1D) ${ }^{22}$. Niche signaling therefore globally acts to prevent GSC differentiation. The C. elegans GSCs, like any other SCs, can therefore be considered as being "primed" for differentiation, essentially waiting for niche signals to weaken to launch their differentiation program.

Variations exist in the way SCs divide and in the molecular nature of niche signaling across species and SC systems. For example, some SCs divide primarily asymmetrically to give rise after each division to another SC and a daughter cell that is bound to differentiate ${ }^{11}$. As such, Drosophila male GSCs divide primarily via asymmetric divisions while hub cells act as their niche by preventing differentiation through activating the JAK/STAT pathway. Drosophila female GSCs also divide asymmetrically, although the niche consists of terminal filament and cap cells that activate TGF- $\beta$ signaling within GSCs ${ }^{23}$. These simple SC model systems showcase a broadly applicable principle regarding the role of niche signaling: to prevent $\mathrm{SC}$ differentiation, which is the default path of all known SCs.

\section{Regulation of stem cell proliferation rates}

When a SC lies within its niche and is thus instructed to remain undifferentiated, it can either grow and divide, or stay still. Growth factors promote SC proliferation, while their absence promotes $\mathrm{SC}$ quiescence, a reversible type of cell cycle arrest. At least two separate kinds of growth factors can stimulate SC growth and proliferation. A systemic signal was shown to stimulate SC proliferation to match its rate to the nutritional status of the animal, such that SCs will tend to proliferate at a higher rate in a well-nourished animal, and at a lower rate in starved animals. A more localized signal further couples the proliferation rates of specific SC populations within an organism to the needs for new differentiated cells in the tissue they serve (Fig. 2A-B). These two mechanisms are individually discussed in the next subsections.

\section{Systemic regulation of stem cell proliferation downstream of nutrition}

Niche signals were rapidly recognized to instruct SC fate, but what controlled the rate of SC proliferation remained unclear until it was noticed that the proliferation of Drosophila GSCs responded to the animal's nutritional status ${ }^{24}$. Indeed, following nutrition, insulin-like peptides are secreted from the animals nervous system and promote GSC proliferation ${ }^{25}$. Nutrition and systemically released insulin-like peptides also support the proliferation of Drosophila intestinal SCs, neuroblasts and hematopoietic progenitors ${ }^{14}$. Equally in C. elegans, insulin-like/IGF-1 signaling (IIS) is activated in response to nutrition and systemically promotes proliferation of their only SC type, GSCs ${ }^{13,26-28}$. In this system, the sole insulin-like receptor DAF-2 inhibits the forkhead transcription factor DAF-16/FOXO autonomously within GSCs to promote their proliferation $^{27,29}$. IIS may similarly systemically support the growth/proliferation of mammalian $\mathrm{SCs}^{14}$, although this remains to be formally demonstrated. 
In C. elegans, when environmental conditions are harsh and/or nutrition is deficient, IIS is reduced and larvae stop development, momentarily sending their GSCs into quiescence, until environmental conditions improve. Several factors are required to properly establish GSC quiescence, downstream or in parallel to IIS, including the dual specificity phosphatase PTEN, and the LKB1/AMPK kinase cascade ${ }^{30-34}$. These proteins are highly conserved and their orthologs have since been reported to similarly influence SC proliferation in mammals. Indeed, PTEN and LKB1 are necessary to maintain murine hematopoietic SC quiescence ${ }^{35-39}$. Exactly how PTEN, LKB1 and AMPK promote GSC quiescence and how their activities may be counteracted by nutrition and IIS remain unclear. Regardless, IIS may act similarly in other organisms and clearly appears as a top candidate for the primary systemic regulator of stem cell proliferation across species ${ }^{13,14}$. However, other systemic factors may also influence the proliferation of certain SC populations, including those linked to immune reponses and circadian rhythms.

\section{Localized regulation of stem cell proliferation by tissue homeostasis}

An animal's nutritional status does not fully explain the proliferative behaviour of all of its SC populations. Indeed, not only do the SCs of each tissue divide at different rates ${ }^{40}$, but a disparity can also be observed across separate populations of the same SC type. In the C. elegans adult hermaphrodite for example, GSC proliferation rates are coupled to nutrition and IIS, but also to the demand for oocytes, their terminally differentiated progenies. When the animal's spermatheca is empty, mature oocytes are no longer fertilized and begin to accumulate in the proximal gonad. This triggers a feedback signal that suppresses GSC proliferation at the distal end, in order to halt the production of new oocytes ${ }^{28,41,42}$. Interestingly, that homeostatic signal is independent within each gonad arm, such that sperm-depletion in one arm does not impair GSC proliferation in the other $\operatorname{arm}^{28}$. Yet, how homeostatic signaling achieves spatial restriction remains unclear.

Regardless, GSC proliferation plummets in sperm-depleted C. elegans hermaphrodites despite sustained feeding and systemically activated IIS, which in fact continues, albeit now inefficiently, to signal GSC proliferation ${ }^{28,29}$. Oocyte accumulation suppresses ERK/MAPK signaling to inhibit GSC proliferation, while that pathway promotes GSC proliferation in parallel to IIS ${ }^{29}$. As such, when young hermaphrodites are grown under replete growth conditions, and their spermathecae contain sperm, IIS acts in concert with ERK/MAPK signaling to promote the high GSC proliferation rates typical of young well-fed adult hermaphrodites (Fig. 2C). If either IIS or ERK/MAPK signaling decreases, GSC proliferation concomitantly decreases, and it completely stalls when both pathways are inactivated simultaneously ${ }^{29}$.

Localized homeostatic SC regulation is probably not restricted to C. elegans. For example, mammalian muscle SCs are normally quiescent throughout adult life, yet they resume proliferation following a muscle injury to participate in repair ${ }^{43}$. Although, to our knowledge, this has not been specifically demonstrated, it is expected that only the muscle SCs located close to the injury resume proliferation. It seems somewhat inconceivable that all muscle SCs across all muscles would reenter proliferation following a localized muscle injury. Therefore, localized homeostatic control of SC proliferation must be a widely-spread phenomenon, applicable to several types of SCs that serve solid tissues such as the brain, lungs and the gastro-intestinal tract. Homeostatic SC regulation also occurs in liquid tissues, such as the blood and lymph of mammals (e.g. homeostatic regulation of erythropoiesis by erythropoietin ${ }^{44}$ ), though it more likely acts systemically within these tissues. 
Future research will be needed to determine whether regulation of SC proliferation by two or more growth factors in parallel is a widely spread phenomenon. In support of that idea however, Drosophila gut intestinal SC proliferation is stimulated both by IIS and homeostatic cytokines released by damaged enterocytes that activate JAK/STAT signaling within intestinal SCs to promote enterocyte replacement ${ }^{14,45}$. Essentially, from the moment SC proliferation has some level of homeostatic control, a second growth factor pathway must combine with, or adjust, IIS regulation. Additional levels of regulation, including from cues related to developmental stage, aging, inflammation, immunity or stress, are likely. Yet, multiple regulatory layers may not apply to all SC populations since GSCs in C. elegans males, which only differentiate into sperm, do not show apparent homeostatic regulation ${ }^{28}$, suggesting that their proliferation may be stimulated solely by IIS.

The mechanisms that enable the homeostatic regulation of SC proliferation to act locally, within a specific tissue, or part of a tissue, remain largely unknown. Yet, the $C$. elegans ERK/MAPK ortholog MPK-1 promotes GSC proliferation cell non-autonomously from the animal's gut or somatic gonad ${ }^{46}$. Therefore, homeostatic signals should, in principle, inhibit or counteract ERK/MAPK signaling within one or both of these tissues in order to suppress GSC proliferation. The gut runs the length of the animals, while each gonad arm is individually enwrapped by somatic sheath cells. Since pathway activity could be controlled independently from each gonad arms, we favor the somatic gonad as the likely site where ERK/MAPK suppression may occur following oocyte accumulation. We predict that such complex regulation, involving multiple cell types, may be common for homeostatic SC proliferation control in other systems.

\section{Niche signaling boundaries confine undifferentiated SCs in vivo}

The SC basic properties and regulatory mechanisms, as defined above, are such that when SCs are isolated from an animal and put in a dish in culture, they essentially require three elements to grow, undergo self-renewing proliferation, and expand in numbers to eventually fill that dish. Let us use the mammalian ESCs to illustrate this point ${ }^{5}$ : ESCs extracted from an embryo need to continue receiving niche signaling in order to remain undifferentiated, otherwise they would launch their default differentiation program. In culture, niche signaling was originally re-created by coating the dish with a layer of inactivated mouse embryonic fibroblasts (MEFs), commonly referred to as "feeder" cells 5 . Nowadays, niche signaling is more conveniently and efficiently replaced by the 2iL cocktail (containing mitogen activated protein kinase kinase (MEK) and glycogen synthase kinase (GSK3) inhibitors (2i), in addition to the cytokine leukemia inhibitory factor (LIF, L)) to the medium, which acts to prevent differentiation and promote the naïve state ${ }^{47-50}$. In order for ESCs to grow and proliferate, they also need basic nutrients to synthesize new nucleic acids and proteins, as well as growth factors to promote nutrient utilization and prevent entry into quiescence. These last two elements are respectively provided by the basic culture medium (e.g. Glascow minimum essential medium, GMEM) and fetal bovine serum (FBS). When provided with these three elements in a dish, ESCs undergo self-renewing proliferation and form a tumour consisting of a mass of SCs; a benign tumour of the undifferentiated type (Fig. 3 Left). These basic principles apply to most other types of SCs that have been successfully cultured, with niche, nutrients and growth factors requiring optimization for each SC type. 
Undifferentiated SC tumours do not normally form in vivo simply because niche signaling is spatially restricted within tissues. Indeed, in a living organism, niche signaling not only ensures SC maintenance, but the size and extent of niche signaling together define a limited space in which SCs can exist. When ESCs expanded in vitro are injected back into a blastocyst, even after several passages, control over their proliferation is re-established in vivo, such that ESC tumours do not usually form following their transplantation ${ }^{5}$. In C. elegans, DTCs have long processes called cytonemes and their extent, along with the level of Notch ligand expression and Notch signaling activation in GSCs, essentially establish the size of the GSC population and $\mathrm{PZ}{ }^{19,22,51}$. As proximal SCs are pushed outside of the niche due to the ongoing proliferation, they enter their default differentiation path, which thereby reduces their proliferation potential. Their ability to initiate an undifferentiated benign tumour thereby becomes highly restricted. Thus, from one standpoint, the niche acts to confine a small undifferentiated SC tumour within a specific region in vivo, essentially preventing it to grow beyond a hard limit. If niche signaling becomes constitutive however, the tumour is deconfined and grows indefinitely (Fig. 3 Left $v s$ right).

As a matter of fact, if niche signaling is upregulated ${ }^{52}$, or if the niche physically expands ${ }^{53}$, the region where proliferative SCs are found expands accordingly, and SC numbers increase. If niche signaling becomes fully constitutive, SCs keep proliferating even when they move outside of the niche and form an ever-expanding benign tumour in vivo, very much like they do in a culture dish (Fig. 3). Spontaneous mutations that activate niche signaling therefore have the potential to induce undifferentiated SC tumours in vivo. In C. elegans for instance, gain-of-function mutations in the Notch receptor GLP-1 induce the formation of undifferentiated GSC tumours (Fig. 4A) ${ }^{52,54}$. Equally, mutations that inactivate genes whose function is necessary for GSC differentiation, can cause the formation of undifferentiated tumours ${ }^{20,55,56}$. In Drosophila male and female germlines, activation of niche signaling, or the inactivation of genes required for GSC differentiation, similarly result in an accumulation of undifferentiated GSCs ${ }^{57-60}$. Therefore, a failure to restrict niche signaling in vivo results in the formation of a benign undifferentiated SC tumour.

Undifferentiated benign tumours are rather seldom found in humans. It is unclear exactly why that is the case, but based on how niche signaling works in simple invertebrates, several reasons may together account for this scarcity. First, germline mutations causing constitutive niche signaling are expected to cause dramatic developmental defects and embryonic lethality. They must therefore be strongly selected against. Second, mutations inducing constitutive niche signaling are typically gain-of-function, which are much less frequent than loss-of-function mutations ${ }^{61}$. In addition, mammalian SC niches and niche signals may be more complex than in invertebrates, and may involve multiple cell types and combinatorial niche signals (e.g. hematopoietic $\mathrm{SCs}^{62}$ ), such that single mutations are unlikely to render niche signaling fully constitutive. Third, loss-of-function mutations in genes that promote differentiation can also cause undifferentiated SC tumours, but these genes typically act redundantly, at least in C. elegans (e.g. lst-1 vs sygl-1; fbf-1 vs fbf-2; gld-1 vs gld-2; gld-3 vs nos-3) ${ }^{20,22,63}$, such that mutations in one single gene have little effects on their own. The chances that two spontaneous hits occur successively in the two (or more) redundant genes in the same SC are very low. This may be particularly true for SCs that follow a model of population asymmetry, since many SCs only divide a number of times before they, along with all of their progeny, are displaced outside of the niche. As such, many SCs may be unable to indefinitely pass on their first hit to an undifferentiated daughter. 


\section{Basic principles of homeostatic stem cell regulation}

If one considers only niche signaling, growth factors and basic nutrients in an animal that is well nourished, SCs would be expected to proliferate at a high rate until the niche is filled, but continue to proliferate at the same rate afterwards, generating a continuous overflow of cells spilling outside of the niche (Fig. 5 Top, Middle). Those cells undergo differentiation and eventually reach a terminal phenotype. For a steady state to exist in the tissue under such optimal growth conditions, the life expectancy $\left(\mathrm{L}_{\mathrm{T}}\right)$ for the terminal cell type $(\mathrm{T})$ has to be inversely proportionate to the maximal rate $\left(\mathrm{R}_{\mathrm{T}}{ }^{\max }\right)$ at which new terminal cells can be produced $\left(\mathrm{L}_{\mathrm{T}}=1 /\left(\mathrm{R}_{\mathrm{T}}{ }^{\mathrm{max}} \mathrm{N}\right)\right.$, where $\mathrm{N}$ represents a nutritional adjustment factor that would be equal to 1 under optimal growth conditions). In other words, for an equilibrium to be maintained, the longer the terminal cells live, the lower the terminal cell production rate needs to be in order to prevent a buildup of terminal cells. Such a simple mode of SC proliferation control, based uniquely on nutrition, would however not easily cope with changing environmental conditions that would perturb either the rate of terminal cell production (e.g. starvation) or terminal cell life expectancy (e.g. tissue injuries). To repair an injury, for example, terminal cells have to be produced faster than they would normally be eliminated in the absence of injury. Most tissues can replace lost cells. However, if terminal cells can be produced faster than they are eliminated (i.e. $\mathrm{L}_{\mathrm{T}}>1 /\left(\mathrm{R}_{\mathrm{T}}{ }^{\mathrm{max}} \mathrm{N}\right)$ ), a buildup of terminal cells would be expected to happen over time in most tissues in well-fed organisms in the absence of injuries (Fig. 5 Left). In other words, if SC proliferation were to depend only on nutrition, all tissues that can regenerate following an injury would grow differentiated benign tumours in wellfed adults in the absence of injuries. To remain balanced at all times, the relation therefore requires another adjustment factor, termed homeostatic $(\mathrm{H})$ regulation. The role of homeostatic regulation is to prevent the buildup of terminal cells in a tissue, which would inevitably happen under optimal growth conditions if terminal cell production was only dependent on nutrition. Accordingly, for all regenerating tissues which do not normally build tumours, $\mathrm{L}_{\mathrm{T}}$ is expected to be smaller than $1 /\left(\mathrm{R}_{\mathrm{T}}{ }^{\max } \mathrm{N}\right)$. The tissue turnover equation is thus corrected by an $\mathrm{H}$-factor as follows: $\mathrm{L}_{\mathrm{T}}=$ $1 /\left(\mathrm{R}_{\mathrm{T}}{ }^{\mathrm{max}} \mathrm{NH}\right)$, such that an equilibrium can be maintained (Fig. 5 Right).

Since the rate of terminal cell production is ultimately dependent on the maximal rate of differentiated progeny production by the underlying stem/progenitor cell population $\left(\mathrm{R}_{\mathrm{SC}}{ }^{\mathrm{max}}\right)$, knowing the relationship between the rate of SC progeny production and terminal cell production allows to predict SC proliferation rates from their terminal cell's life expectancy at equilibrium, and vice-versa. Namely, $\mathrm{R}_{\mathrm{T}}{ }^{\mathrm{max}}=\mathrm{XR}_{\mathrm{SC}}{ }^{\mathrm{max}}$, where $\mathrm{X}$ is a conversion factor specific to each $\mathrm{SC}$ type: terminal cell type pair, and $\mathrm{L}_{\mathrm{T}}=1 /\left(\mathrm{R}_{\mathrm{T}}{ }^{\mathrm{max}} \mathrm{NH}\right)=1 /\left(\mathrm{XR}_{\mathrm{SC}}{ }^{\mathrm{max}} \mathrm{NH}\right)$. During development or following an injury, the $\mathrm{H}$ value may approach 1, allowing SCs to increase proliferation (Fig. 6A-C). This pushes the actual rate of terminal cell production towards $\mathrm{R}_{\mathrm{T}}{ }^{\mathrm{max}}$ and promotes tissue repair. After completion of the repair, the $\mathrm{H}$-factor must decrease back to its equilibrium value to prevent the overproduction of SC progeny. Conversely, if conditions permit an increase in terminal cell life expectancies, the H-factor must increase to slow down SC proliferation and prevent terminal cell buildup (Fig. 6D). Finally, a mutation preventing homeostatic regulation would disconnect SC proliferation from tissue needs, resetting to their maximal proliferation rate (as now only governed by nutrition), as during development or a repair situation. However now, in absence of the extra space left either for tissue expansion during development, or to replenish lost cells during tissue 
repair, the extra terminal cells produced now start to pile up, forming a differentiated benign tumour (Fig. 6E).

\section{The documented case of $C$. elegans}

Let us use the $C$. elegans germline to illustrate the reach of these principles. In adult hermaphrodites, GSCs proliferate to differentiate exclusively into oocytes. This occurs essentially in a linear assembly line-like fashion (Fig. 1), which allows to easily measure equation parameters. When sperm and food are not limiting, each gonad arm ovulates a new oocyte every 24 minutes on average $\left(\mathrm{R}_{\mathrm{T}}{ }^{\max }=2.5\right.$ oocytes/hour $)$, translating into $\sim 5$ eggs laid/hour ${ }^{64,65}$. Although there are usually 5-6 terminal (diakinesis) stage oocyte lined-up in the proximal part of the gonad $\operatorname{arm}^{28,29,66}$, we consider only the proximal-most (-1) oocyte as the terminal cell type since it is the only one that can be ovulated, and thus "exit" the tissue, at any time. To ensure the production of 1 oocyte every $\sim 24$ minutes, a pool of $\sim 130$-160 GSCs divide every 6-8 hours at the distal end of the gonad, for an output of $\sim 20 \mathrm{GSC}$ progenies/hour ( $\mathrm{R}_{\mathrm{SC}}{ }^{\mathrm{max}}=20 \mathrm{GSC}$ progenies/hour) exiting the niche $^{67}$. A large difference exists between GSC progeny ( $\mathrm{RSC}^{\max }=20 \mathrm{GSC}$ progenies/hour) and oocyte production ( $\mathrm{R}_{\mathrm{T}}{ }^{\max }=2.5$ oocytes/hour), largely because more than half of germ cells die by apoptosis during meiosis to serve as nurse cells, contributing their cytoplasm to the growing oocytes via cytoplasmic streaming in a central rachis to which all germ cells are connected ${ }^{68,69}$. Based on these numbers, we can estimate that roughly 8 GSC progenies are necessary to give rise to one oocyte. The $\mathrm{R}_{\mathrm{T}}{ }^{\mathrm{max}}$ to $\mathrm{R}_{\mathrm{SC}}{ }^{\mathrm{max}}$ conversion factor $(\mathrm{X})$ can thus be estimated as $1 / 8$ or $\sim 0.125$ oocyte/GSC progeny. The introduction of conversion factors may not be necessary in all SC systems, for example if apoptosis is not significant, but may be required if SCs can differentiate into multiple different terminal cell types.

The above stands when animals are fed ad libidum, and the nutrition factor is equal to 1 . However, when animals lack food, IIS is reduced and GSC proliferation drops towards zero as animals eventually enter adult reproductive diapause in the complete absence of food ${ }^{70}$. In that direction, a strong inactivation of the insulin/IGF-1 receptor DAF-2 lowers larval and adult GSC proliferation $^{27,28}$. It will be necessary to evaluate ovulation rates and/or niche outputs under various conditions to evaluate nutrition factor $(\mathrm{N})$ dynamics.

When well-fed hermaphrodites $(\mathrm{N}=1)$ run out of sperm, or in feminized mutants that do not make sperm (e.g. fog-1, fog-2 $)^{41,71}$, terminal stage oocyte life expectancy dramatically increases because their final maturation and ovulation is only provoked by a cascade downstream of sperm-released major sperm proteins (MSPs) ${ }^{72}$. Thus, in principle, they should arrest in meiotic prophase indefinitely until sperm is re-supplied. In the absence of sperm, oocyte ovulation rate was measured at $<0.1$ ovulation/hour/gonad $\mathrm{arm}^{65,73}$. As a result, terminal stage oocytes initially pile up in the proximal gonad (Fig. 2) until homeostatic signaling downregulates GSC proliferation and meiotic progression, stalling the whole assembly line. While the homeostatic response is generated, the number of terminal-stage oocytes in the proximal gonad increases from $\sim 5-6$ to more than 15 , while oocytes get obviously compressed against each other as they pile up ${ }^{28,72,73}$. In response, GSC proliferation drops at the distal extremity of the gonad ${ }^{28,29}$, such as to balance the increased terminal oocyte life expectancy, and reaches a new low-turnover homeostatic equilibrium. Since the actual ovulation rate is lower than 0.1 ovulation/hour/gonad at this new equilibrium, the life expectancy of the -1 oocyte must be greater than 10 hours. The lifespan of a diakinesis oocyte in a spermless hermaphrodites after having stacked 15 oocytes must then exceed 
150 hours $(>10$ hours $\times 15$ oocytes $)$. Solving $\mathrm{L}_{\mathrm{T}}=1 /\left(\mathrm{R}_{\mathrm{T}}{ }^{\mathrm{max}} \mathrm{NH}\right)$ for a well-fed spermless hermaphrodite using $\mathrm{L}_{\mathrm{T}}>10$ hours yields $\mathrm{H}<0.04$. Using an output of less than 0.1 oocyte/hour and a $C$. elegans-specific X conversion factor of 0.125 oocyte/GSC progeny, we can estimate that the niche produces less than 0.8 GSC progenies/hour in spermless hermaphrodites.

\section{Homeostatic SC regulation prevents differentiated benign tumours}

Mutations in genes encoding several highly conserved proteins prevent homeostatic GSC regulation in $C$. elegans. These include loss-of-function in DAF-18/PTEN, PAR-4/LKB1 and AAK-1/AMPK, and a gain-of-function in LET-60/Ras ${ }^{28,29}$. How these genes work together to maintain germline homeostasis is not yet fully understood. However, they must join in a multistep process that implicates not only the SCs and their terminally-differentiated progeny, but also cells of unrelated origins, within the somatic gonad. In this system, what appears to generate a homeostatic signal is an accumulation of oocytes, and for this to happen, oocytes need to arrest in the absence of induction by sperm. This step fails in DAF-18/PTEN, PAR-4/LKB1 and AAK1/AMPK loss-of-function mutants, as their oocytes spontaneously activate in the absence of sperm signals, are ovulated, and laid as endomitotic oocytes ${ }^{28,29}$. In this case, there is no terminal cell buildup because unfertilized oocytes are ovulated and laid essentially as quickly as if they were fertilized and laid as eggs; their life expectancy remaining short. However, when the spontaneous activation of oocytes is blocked in aak-1/AMPK mutants by the removal of OMA-1 and OMA-2, two zinc-finger proteins redundantly required for oocyte activation ${ }^{74}$, the deleterious effects of homeostatic signaling failure are far more dramatic. In such animals, GSCs keep proliferating despite the ongoing proximal accumulation of oocytes and a large benign tumour that largely consists of differentiated oocytes, rapidly forms (Fig. 4B) ${ }^{29}$. Arrested oocytes occur in multiple rows in the proximal gonad, inside the uterus, and sometimes break open the germline basal membrane to venture into interstitial fluid, likely due to the resulting increase in intra-organ pressure.

Differentiated benign tumours therefore arise when homeostatic signaling is disrupted (Figs. 5,6E). Within tissues in which the terminal cell type is continually expulsed or desquamated at a fast rate, even if SC proliferation were to remain maximal at all times, a benign tumour may not form because terminal cells have a short life expectancy, roughly equal to the inverse of the their maximal production rate (e.g. C. elegans oocytes when sperm is present, or when sperm is absent but DAF-18/PTEN, PAR-4/LKB1 or AAK-1/AMPK are lost). If that life expectancy increases but is not shortly counterbalanced by a decrease in oocyte production (itself enabled by a decrease in GSC proliferation), a benign tumour is expected to grow.

To extrapolate what such a relationship could mean in other tissues and organisms, we can use the mammalian intestinal epithelium as an example. This epithelium consists of several types of differentiated cells, including enterocytes, goblet and tuft cells, which are generated following the differentiation of transit amplifying cells, themselves generated following intestinal SC (ISCs) proliferation and differentiation ${ }^{75}$. The differentiated enterocytes cells that make the inner lining of our intestinal tract have a relatively short life expectancy and must be replaced every 5-7 days, on average, and to replace them, ISCs divide once a day, a relatively high rate, throughout adulthood $^{76}$. The lifespan of an intestinal enterocyte is likely affected by multiple factors, including diet, nutritional status, inflammation and microbiome constitution. Indeed, inflammatory bowel conditions are known to accelerate gastrointestinal (GI) epithelial lining desquamation ${ }^{77}$, while a 
range of ingested substances, from irritant laxatives (e.g. bisacodyl) to red meat, may also have similar, perhaps more graded, effects ${ }^{78,79}$. As such, one would expect ISCs to proliferate more in individuals with high GI lining turnover. On the other hand, anti-inflammatory treatments may lead to an increase in GI epithelial cell life expectancy in these individuals, while ISC proliferation is expected to decrease accordingly. Assuming such homeostatic regulation takes place in the human GI tract, as in the Drosophila intestine ${ }^{45,80}$, its disruption is expected to cause the formation of benign intestinal tumours. Such benign tumours would originate from misregulated ISCs, while these misregulated ISCs would show increased proliferation relative to homeostatically-regulated ISCs, and would thus have increased chances of gaining new genetic and epigenetic changes and of further evolving towards cancer. In support of this view, it was proposed that intestinal cancers originate from ISCs that, through acquiring changes, escaped from normal regulation ${ }^{81}$, while patients inflicted with inflammatory bowel diseases have a greater risk of developing intestinal cancers $^{82}$.

\section{How benign tumours may occur and evolve towards cancer}

Once an individual's reproductive period is over, the effect of natural selection at the organismal level greatly weakens ${ }^{83}$ and as a result, the natural selection occurring at the cellular scale gains freedom. At the cellular level, natural selection favours cell proliferation and survival, eventually leading to cancer, which is, unfortunately for the organism, the ultimate fitness status for a cell: immortality and an unlimited proliferation potential. Ironically, many widely-used cancer cell lines have won the evolution lottery by getting adopted for laboratory research (e.g. HeLa cells) and are now propagated in multiple laboratories around the globe, having truly achieved immortality.

Cancer-initiating mutations are more likely to arise in SCs since they persist and divide throughout our lives. In contrast, their differentiated progenies all have limited life expectancies and/or proliferation potentials, reducing their evolutionary prospectives ${ }^{40,84,85}$. As such, SCs uniquely have a high risk of accumulating genetic and epigenetic changes over the years, and of "evolving" towards cancer. Although this may be less common, early progenitors can undergo transforming mutations conferring them immortality, and can also continue to evolve towards cancer thereafter ${ }^{86,87}$.

Benign differentiated tumours should therefore typically develop after a SC acquires changes that disrupt homeostatic SC regulation. This could occur, for example, following changes that would prevent proper SC differentiation, such that an accumulation of poorly differentiated SC progeny would no longer induce SC quiescence (Fig. 7A). In this case, mutations altering terminal differentiation could promote the formation of benign, partially or aberrantly differentiated tumours. As a result, these mutations favour SC proliferation and at the same time, they increase SC mutation acquisition rates, and thus accelerate their evolution towards cancer. In systems in which homeostatic regulation involves multiple tissues originating from different SC types, it is possible to imagine situations where deregulation within one tissue would nonautonomously promote the formation of a benign tumour in another tissue (Fig. 7B). 


\section{Hamartomatous Polyposis Syndromes offer potential model validation}

In humans, hemizygous germline mutations in PTEN or LKB1 predispose carriers to Cowden's and Peutz-Jegher's syndromes, respectively, which are characterized by the appearance of multiple benign tumours, commonly arising in the gastrointestinal tract, skin and mucous membranes, with an age of onset typically between 10 and 30 years old ${ }^{88,89}$. The tumours, called hamartomas, are benign overgrowth largely consisting of differentiated cells ${ }^{90}$. In addition, these patients have a greatly increased lifetime risk for multiple cancers, including GI cancers.

ERK/MAPK is an oncogene that is aberrantly activated in many different types of cancers, including those of the GI tract ${ }^{91}$. Incidentally, work in the C. elegans germline has shown that PTEN and LKB1 are required for inhibiting ERK/MAPK signaling during homeostatic stem cell regulation ${ }^{29}$. PTEN and LKB1 thus prevent the formation of benign tumours in the C. elegans germline by inhibiting GSC proliferation when their terminally-differentiated progenies are in excess. If some of these principles were conserved in humans, it could explain, at least in part, how Cowden and Peutz-Jegher's patients develop benign tumours, from a problem in homeostatic SC regulation, potentially compromising the downregulation of ERK/MAPK signaling when there are enough differentiated cells in the tissue.

In principle, the apparition of hamartomas in Cowden and Peutz-Jegher's patients could directly result from PTEN and LKB1's hemizygosity. Tumours typically begin to appear in patients around the onset of adulthood, or after development, when the effect of reduced homeostatic signaling is expected to become more apparent. A subtle reduction in homeostatic signaling, such as one caused by hemizygosity, would have little consequence during development since tissues are generally expanding, but the effect would become apparent during adulthood, when tissues stop expanding and homeostatic signaling has to be more robust. As such, it may be worth assessing whether a mild inhibition of ERK/MAPK signaling in Cowden's and PeutzJegher's patients could decrease or delay hamartoma incidence, and perhaps even lower cancer risk $^{92,93}$.

The increased lifetime cancer risk of these patients could then be explained by the increase in proliferation of these patient's SCs in every tissue where homeostatic regulation relies on PTEN and/or LKB1. Their SCs dividing more frequently than average, they are indeed more likely to experience a greater number of genetic and epigenetic changes, which, combined with natural selection, are expected to drive overproliferating SCs towards cancer faster and faster, in a vicious circle.

\section{Perspectives}

Although key tumour suppressors and oncogenes such as PTEN and ERK/MAPK hitherto appear to play a conserved role in homeostatic signaling across different organisms and stem cell types, the hypothesis presented here remains largely speculative and would require further mechanistic validation in vertebrate systems. A range of additional pathways may also be involved in these more complex SC systems. Interestingly, ERK/MAPK promotes SC proliferation cell nonautonomously in $C$. elegans, from a neighboring tissue ${ }^{46}$. PTEN and LKB1 may therefore also work cell non-autonomously to inhibit ERK/MAPK in this tissue. Homeostatic regulation may similarly involve several cell types in humans, and it will certainly be a challenge to solve it down 
to the molecular level for every SC systems. However, the resulting knowledge should greatly facilitate cancer prevention and treatment, as well as all SC-based therapies.

\section{Acknowledgements}

We thank Alexandre Clouet, Pier-Olivier Martel, Jichao Deng, Geneviève Pépin, Alexandre Fisette and Jean-Claude Labbé for helping us improve the manuscript. Research in the Narbonne Laboratory is funded by grants from the FRQ-S (265445), NSERC (RGPIN-2019-06863, RGPAS2019-00017, DGECR-2019-00326) and the CIHR (PJT-169138) to PN. PN is an FRQ-S Junior 1 Bursary Scholar (252405) and holds a UQTR research chair. 


\section{Figure and Legends}

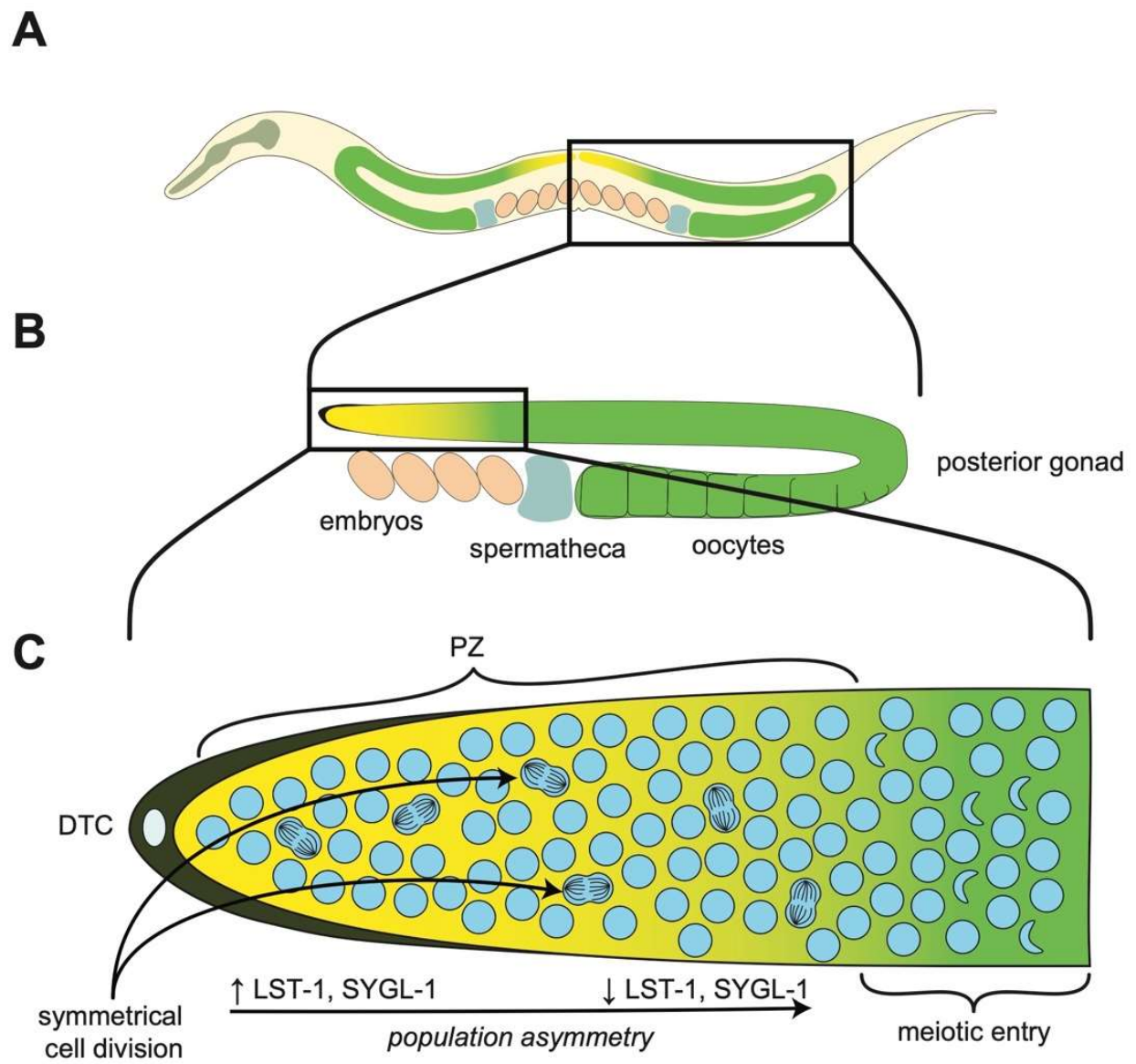

D

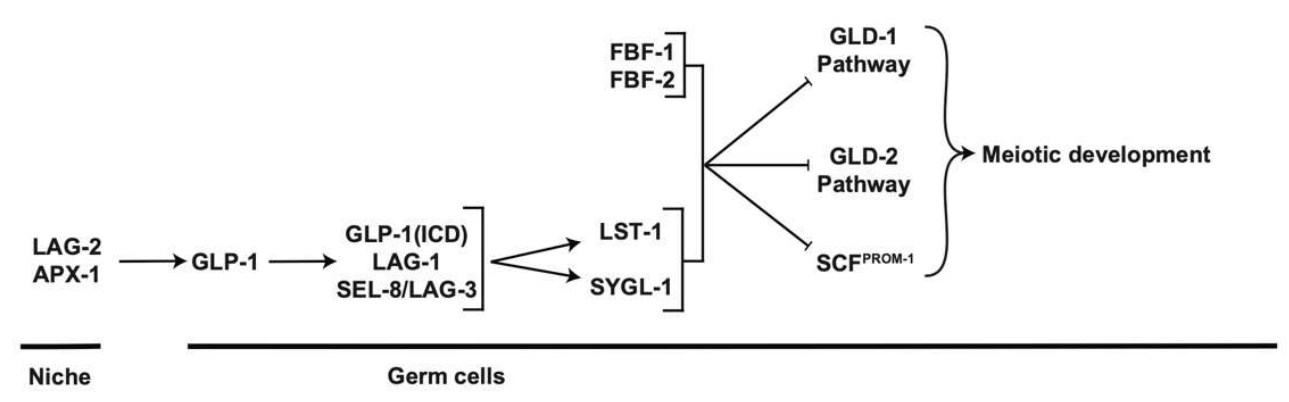

Figure 1. The C. elegans GSC system. (A) Diagram of an adult $C$. elegans hermaphrodite, highlighting its two symmetrical U-shaped gonad arms (adapted from www.wormatlas.org). (B) Magnified schematic of the animal's posterior gonad arm. (C) Further magnified schematic of the distal part of the gonad, revealing the niche cell (DTC; black), the progenitor zone (PZ; yellow), and meiotic entry zone (green). GSCs undergo symmetric divisions as the two daughters have an equal fate potential. Yet, a population asymmetry becomes apparent through a gradient of GLP-1 
activity, and levels of its transcriptional targets LST-1 and SYGL-1, that fades proximally, until a threshold is reached and cells commit to meiotic entry ${ }^{18-21}$. This is apparent as their nuclei adopts the shape of a crescent. (D) Simplified genetic model for niche signaling. The DTC acts as the GSC niche by expressing the Notch ligands LAG-2 and APX-1, which activate the receptor GLP1 at the surface of GSCs. When GLP-1 is activated, its intracellular domain (ICD) is cleaved and serves as a co-activator for the transcription factor LAG-1, which together with LAG-3 and SEL8 , binds to and activates the transcription of two main target genes, lst- 1 and sygl-1 ${ }^{18-21}$. LST-1 and SYGL-1 then work together with FBF-1 and FBF-2, to repress three largely parallel, but partly redundant pathways (GLD-1, GLD-2, and $\mathrm{SCF}^{\mathrm{PROM}-1}$ ) that together promote differentiation. Simplified from ${ }^{22}$.
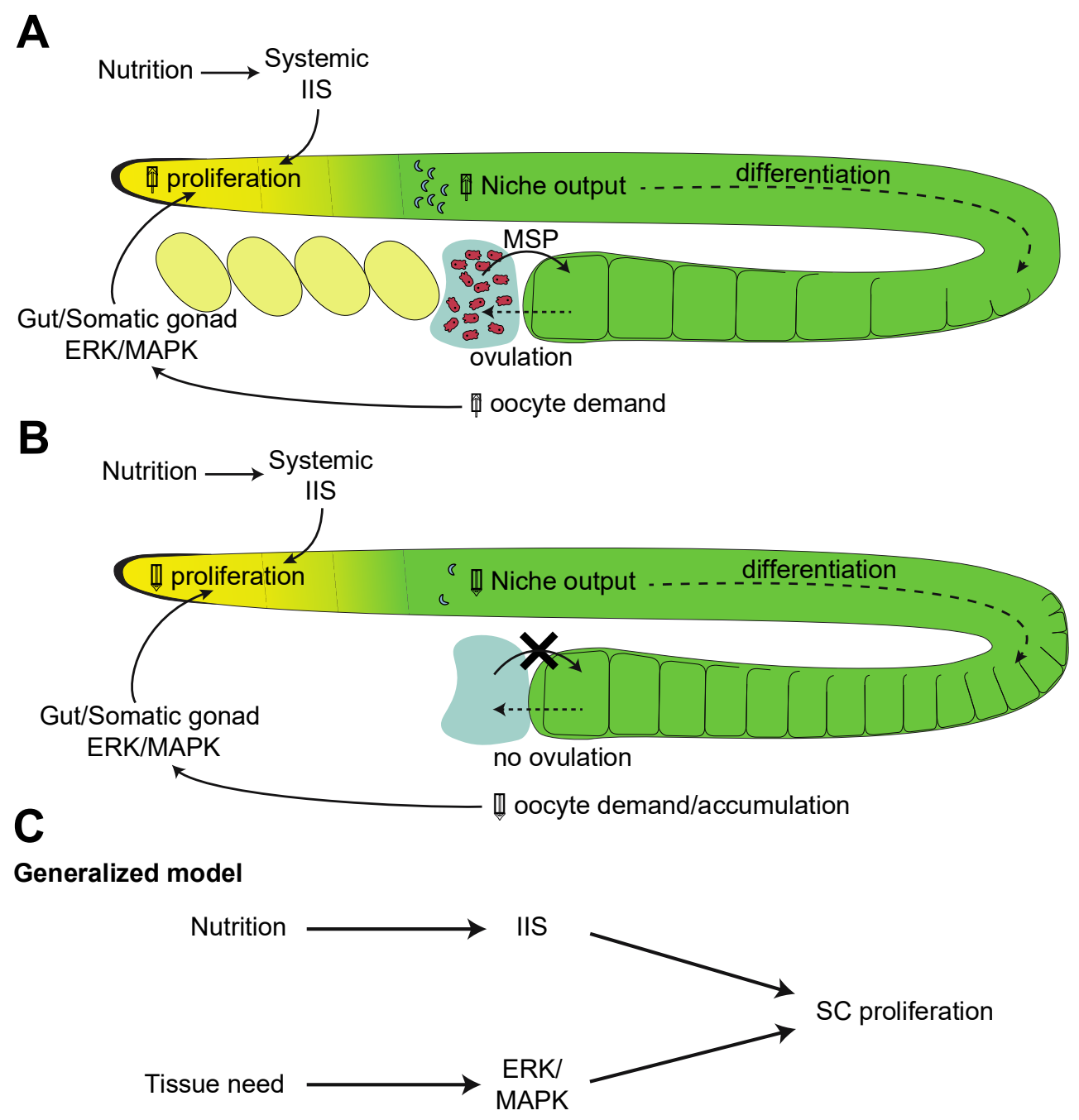

Figure 2. SC proliferation regulatory mechanisms. (A) Schematic representation of the two known mechanisms that promote GSC proliferation in young adult C. elegans hermaphrodites. The nutritional status systemically regulates GSC proliferation through activating insulinlike/IGF-1 signaling (IIS). In this case, the insulin receptor DAF-2 and its main downstream transcriptional target DAF-16/FOXO both act within the germline to influence GSC 
proliferation ${ }^{27,29}$. In parallel, the presence of sperm, through release of major sperm proteins (MSP), promotes oocyte ovulation, maintaining a high oocyte demand. Through an unknown mechanism, this leads to the activation of MPK-1/ERK in the animal's gut and/or somatic gonad, non-autonomously promoting GSC proliferation, sustaining an elevated niche output and rapid oocyte production. (B) When sperm is depleted, ovulation is suppressed and oocytes initially accumulate in the proximal gonad, thereby reducing the demand for more oocytes. This is hypothesized to suppress MPK-1/ERK signaling in the gut and/or somatic gonad, thereby slowing down GSC proliferation, and consequently niche output and oocyte production. (C) A simplified and generalized model for the regulation of SC proliferation by nutrition and tissue demand. Note that while the function of IIS thus far appears well-conserved, ERK/MAPK signaling could be replaced by other pathways in different $\mathrm{SC}$ types or organisms. Other regulatory mechanisms are also possible. 


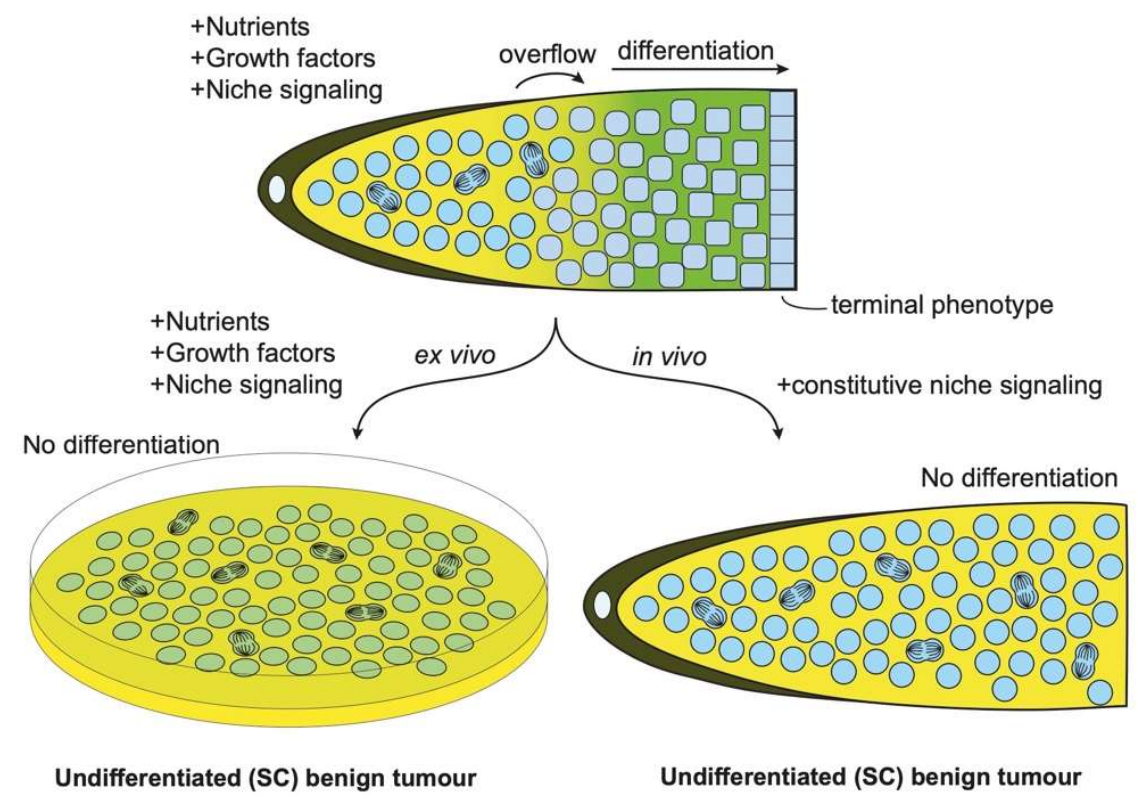

Figure 3. Undifferentiated SC tumours in vitro and in vivo. (Top) In vivo, SCs are maintained in the undifferentiated state and allowed to proliferate within a physical niche (dark gray). Nutrients and growth factors can promote SC proliferation within the niche, but proliferation within a restricted space produces an overflow of SC progeny that exits the niche and embarks on the differentiation path, until they reach a terminal phenotype to function within a tissue. (Bottom left) When SCs are extracted from an animal and grown in vitro in a dish, they require the same three factors to be added within the culture medium to keep proliferating. SCs then form an everexpanding undifferentiated benign tumour that can be passed on indefinitely. (Bottom right) In vivo, undifferentiated benign tumours occur when a mutation constitutively activates niche signaling. In such situation, SCs remain undifferentiated even when they exit the physical niche and proliferate to fill up the entire tissue. The same result can happen if mutations prevent SC differentiation (not shown). 

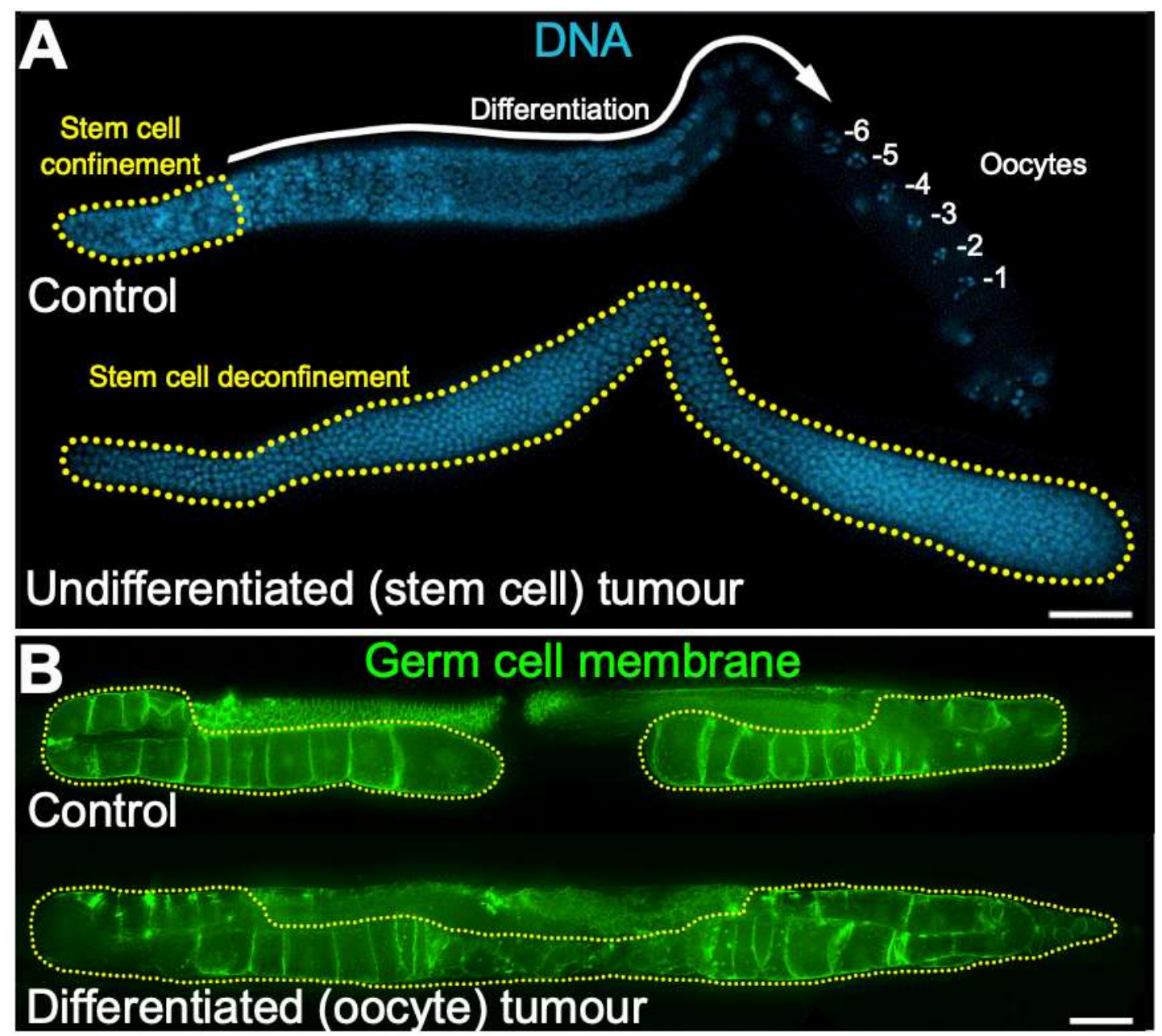

Figure 4. Undifferentiated and differentiated benign germline tumours in C. elegans. (A) DAPI-stained dissected germlines from (top) wild-type and (bottom) $g l p-1$ (gf) hermaphrodites. Undifferentiated mitotic germ cells (yellow dotted lines) are confined within the niche in the wildtype, while their progenies that exit the niche enter meiosis and differentiate into oocytes. Undifferentiated germ cells are completely deconfined and fill the entire gonad in $g l p-1(g f)$ mutants. $g l p-1$ (gf) exact genotype was $d p y$-19(e1259) unc-32(e189) glp-1(oz112gf)/dpy-19(e1259) unc-32(e189) glp-1(oz112gf); qDp3 (qDp3 contains unc-32(e189) and wild-type copies of $d p y$ 19 and $g l p-1)$ ). Distal, left. Scale bar, $20 \mu \mathrm{M}$. Reproduced with permission ${ }^{94}$. (B) Whole (top) control and (bottom) aak-1(tm1944) mutant hermaphrodites having their germ cell membranes labelled with monomeric NeonGreen. When oocytes are not fertilized in control animals, they accumulate up to a certain point (yellow dotted lines), after which homeostatic signaling limits any further oocyte production. In mutants that disrupt homeostatic signaling however, such as aak$1(\varnothing)$, oocyte production does not stop and differentiated benign tumours develop. Anterior, left; dorsal, up. Genotypes include cpSi42[Pmex-5::mNeonGreen::PLC - -PH::tbb-2 3'UTR + unc119(+)]; oma-1(zu405te33); oma-2(te51). Scale bar, $50 \mu \mathrm{M}$. Reproduced with permission ${ }^{29}$. 
+ Nutrients + Growth factors

+ Niche signaling

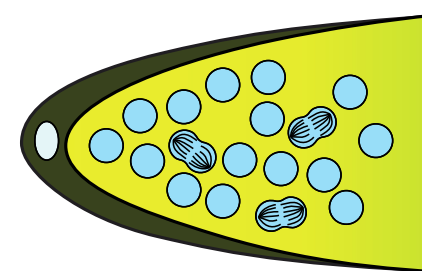

4 proliferation

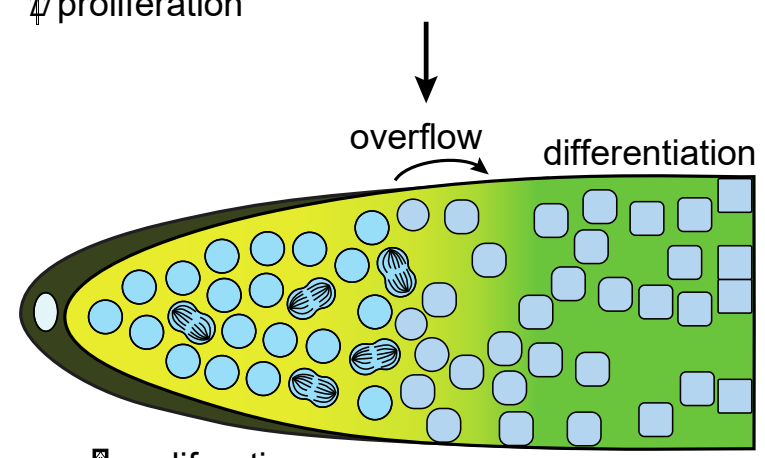

更 proliferation

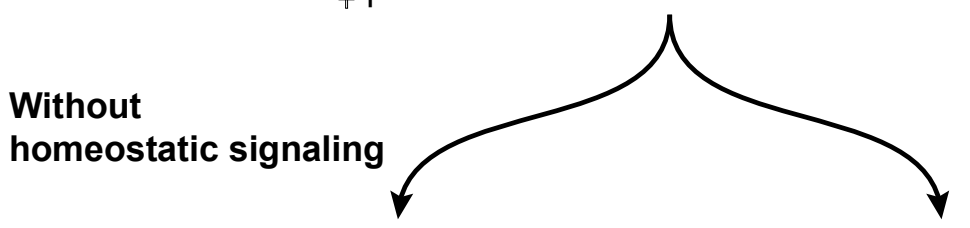

With

homeostatic signaling
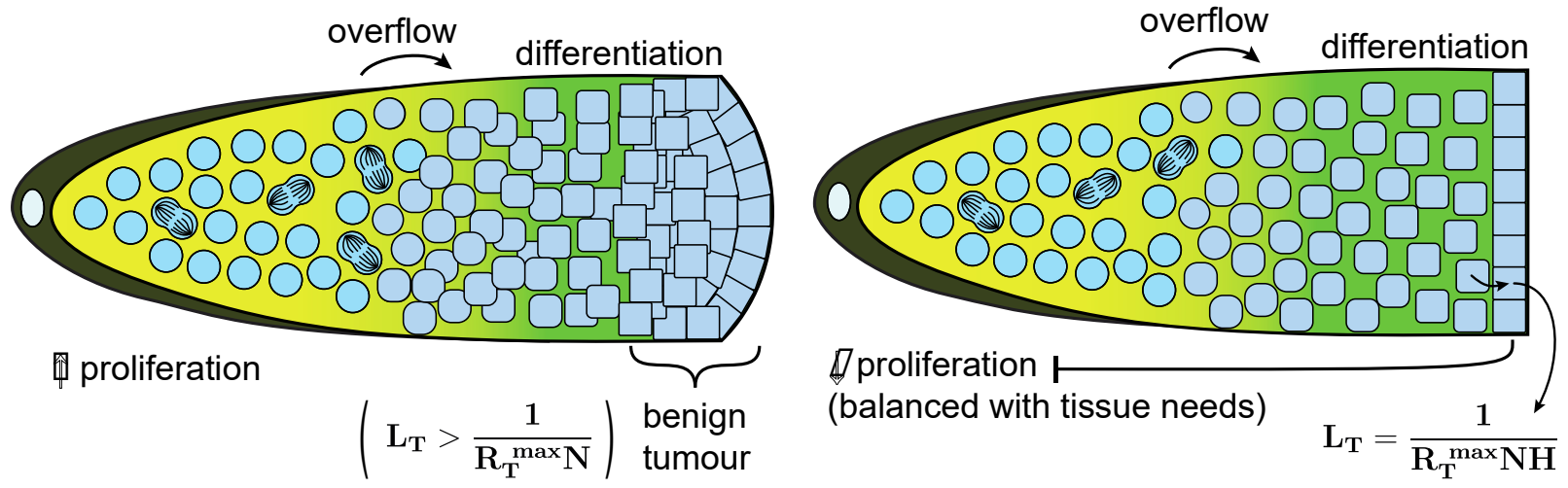

Figure 5. Homeostatic signaling prevents formation of differentiated benign tumours. (Top) If SCs are exposed to niche signaling, are stimulated by growth factors, and receive sufficient nutrients, they will proliferate to expand in numbers. (Middle) When the niche is filled with SCs, they continue to proliferate because they still receive nutrients and growth factors, but some begin to overflow outside of the niche and those undertake the differentiation route, morphing into a terminal cell type. (Bottom left) In the absence of homeostatic signaling, SC proliferation continues at full capacity at all times, even if the tissue they serve has too many terminal cells, causing the formation of a differentiated benign tumour. (Bottom right) When the tissue has enough terminal cell type to fulfil function, homeostatic signaling allows to tune down SC proliferation and the production of new terminal cells. 


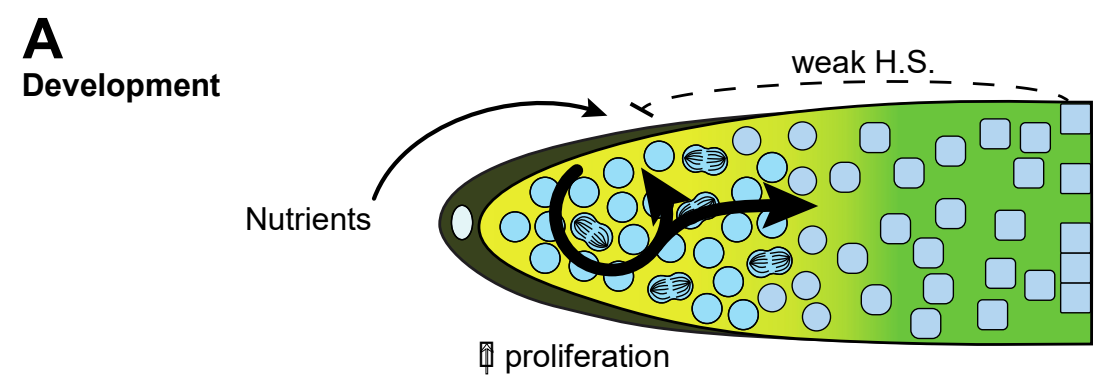

B

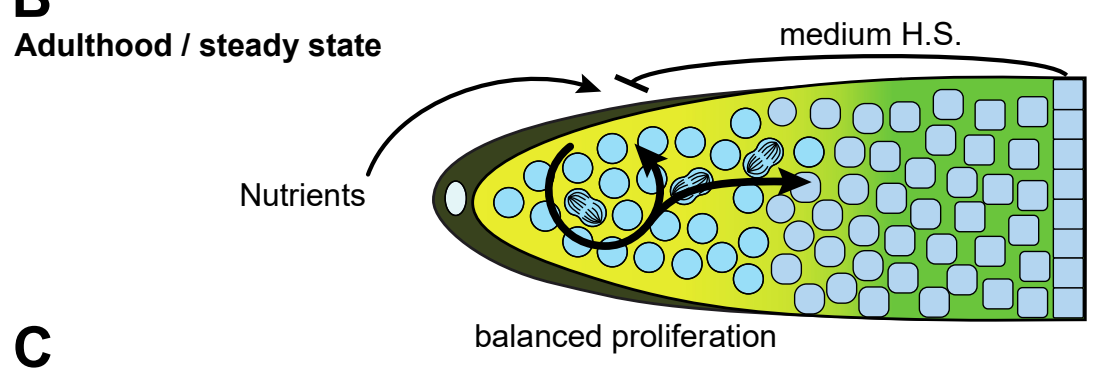

Wound healing

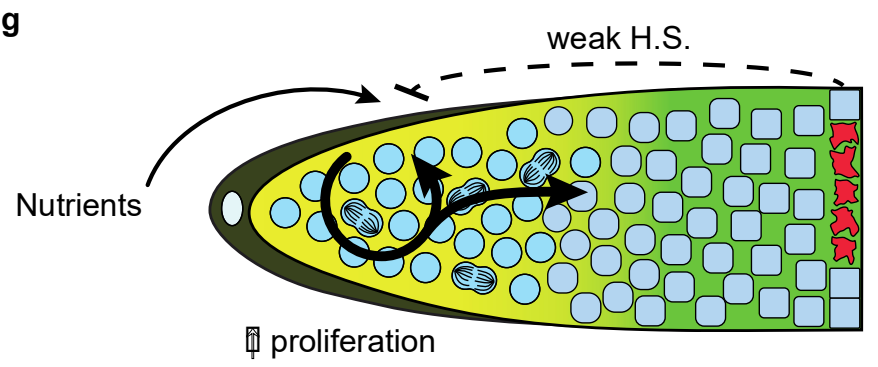

D

Reduced turnover

high H.S.

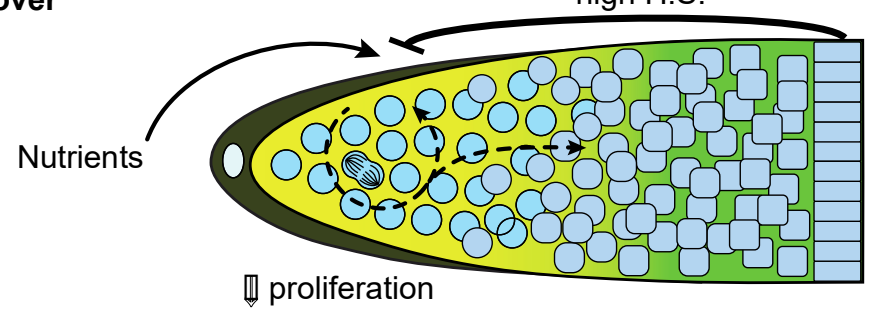

E

Mutation inactivating H.S. mutation inactivating H.S.

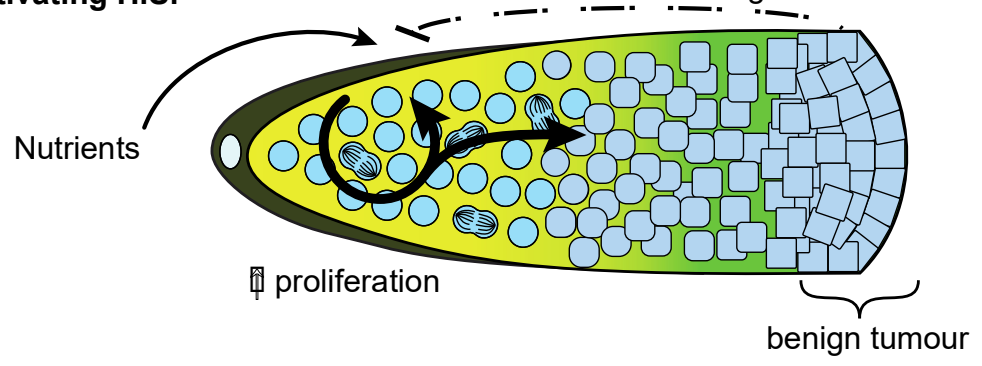

Figure 6. Homeostatic signaling during development and adulthood. (A) During development, tissues are generally expanding in size and require growing numbers of SC progenies. The effects of homeostatic signalling are therefore likely reduced, favouring sustained elevated SC proliferation and differentiation rates. (B) During adulthood, tissues stop expanding and a steady 
state is established between SC proliferation and terminal cell life expectancy. Homeostatic signaling strength is increased just enough to suppress excessive $\mathrm{SC}$ proliferation and arrest tissue growth. (C) A wound causing the loss of terminal cells within a tissue is however expected to reduce homeostatic signaling strength, so that $\mathrm{SCs}$ can increase proliferation to return towards elevated developmental rates to promote repair. (D) In contrast, if for environmental reasons (e.g. a change in life habits - something equivalent to sperm depletion for C. elegans GSCs) terminal cell turnover is reduced (i.e. terminal cell life expectancy is increased), homeostatic signaling strength increases to further suppress SC proliferation and prevent the formation of a benign differentiated tumour. (E) A germline mutation (represented by the darker shade) disrupting homeostatic signaling in a tissue would be equivalent to pursuing a developmental regime of elevated SC proliferation into adulthood, inevitably leading to the production of an excess of differentiated progenies, leading to their accumulation and the formation of a benign tumour. 
A

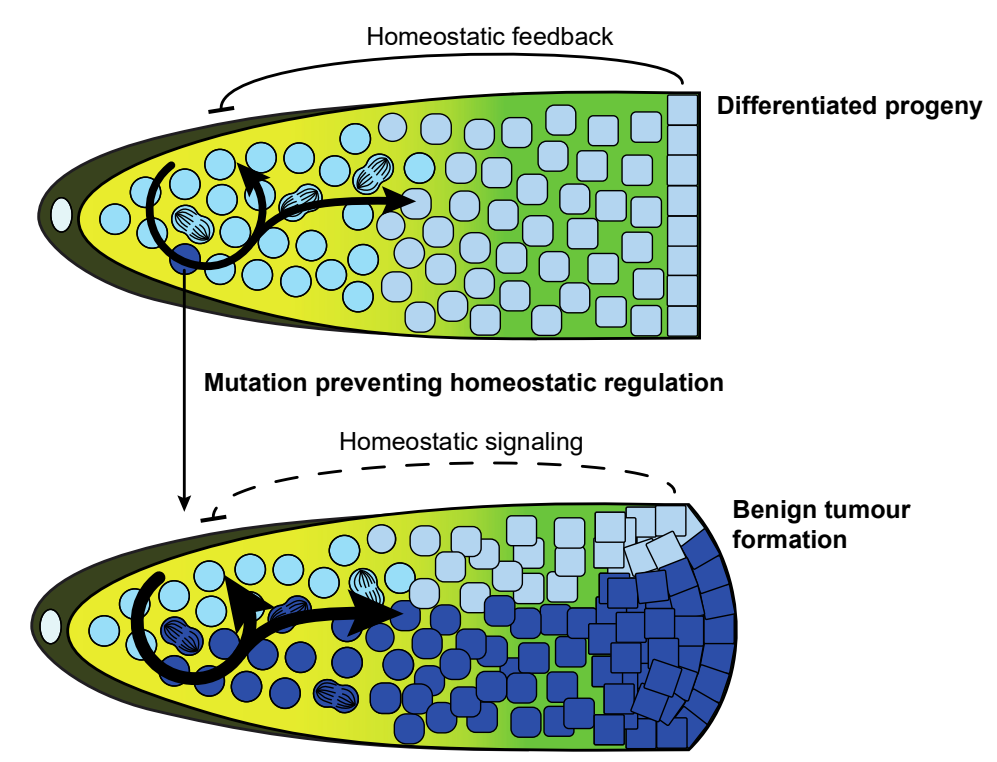

B

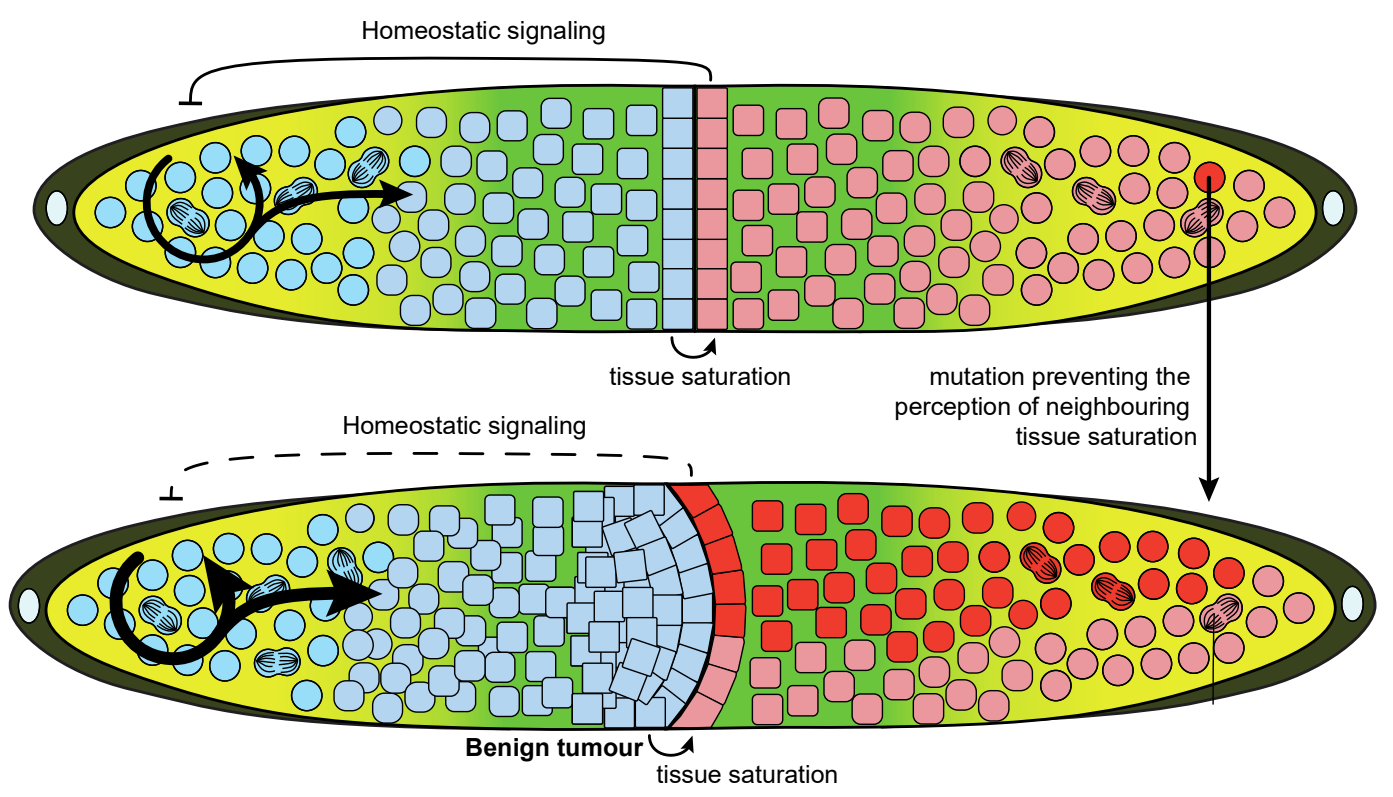

Figure 7. Potential routes towards tumorigenesis and cancer. (A) When a mutation preventing homeostatic signaling occurs in a SC (represented by a darker shade), it is clonally transmitted to both its SC and differentiated progenies. Terminally differentiated cells from such clone no longer 
generate a homeostatic feedback, resulting in enhanced SC proliferation and the formation of a benign tumour. The clone gains an indirect proliferative advantage and the increase proliferation accelerates its evolution towards cancer. (B) In situations in which terminal cell saturation for a given tissue (blue) would be perceived by a neighboring unrelated tissue (red), a mutation (or other changes in tissue status, such as stress or inflammation levels) preventing the perception of blue tissue saturation in a red SC (darker shade) could, in principle, allow for a benign tumour to form in the neighboring tissue, conferring a proliferative advantage to blue SCs. Several other situations are possible. 


\section{References}

1 Clark, W. H. Tumour progression and the nature of cancer. Br J Cancer 64, 631-644 (1991).

2 Qin, H. et al. The role of stem cells in benign tumors. Tumour Biol, doi:10.1007/s13277016-5370-x (2016).

3 Skoric, T. et al. Clinical and morphological features of undifferentiated monomorphous GH/TSH-secreting pituitary adenoma. Eur J Endocrinol 140, 528-537, doi:10.1530/eje.0.1400528 (1999).

4 Becker, A. J., Mc, C. E. \& Till, J. E. Cytological demonstration of the clonal nature of spleen colonies derived from transplanted mouse marrow cells. Nature 197, 452-454 (1963).

5 Evans, M. J. \& Kaufman, M. H. Establishment in culture of pluripotential cells from mouse embryos. Nature 292, 154-156 (1981).

6 Gairdner, R. L. \& Papaioannou, V. E. in The early development of mammals 107-132 (Cambridge University Press, 1975).

$7 \quad$ Schofield, R. The relationship between the spleen colony-forming cell and the haemopoietic stem cell. Blood Cells 4, 7-25 (1978).

8 Hardy, R. W., Tokuyasu, K. T., Lindsley, D. L. \& Garavito, M. The germinal proliferation center in the testis of Drosophila melanogaster. J Ultrastruct Res 69, 180190 (1979).

9 Wieschaus, E. \& Szabad, J. The development and function of the female germ line in Drosophila melanogaster: a cell lineage study. Dev Biol 68, 29-46 (1979).

10 Kimble, J. E. \& White, J. G. On the control of germ cell development in Caenorhabditis elegans. Dev Biol 81, 208-219 (1981).

11 Morrison, S. J. \& Kimble, J. Asymmetric and symmetric stem-cell divisions in development and cancer. Nature 441, 1068-1074, doi:10.1038/nature04956 (2006).

12 Morrison, S. J. \& Spradling, A. C. Stem cells and niches: mechanisms that promote stem cell maintenance throughout life. Cell 132, 598-611, doi:10.1016/j.cell.2008.01.038 (2008).

13 Narbonne, P. \& Roy, R. Regulation of germline stem cell proliferation downstream of nutrient sensing. Cell Div 1, 29, doi:10.1186/1747-1028-1-29 (2006).

14 Shim, J., Gururaja-Rao, S. \& Banerjee, U. Nutritional regulation of stem and progenitor cells in Drosophila. Development 140, 4647-4656, doi:10.1242/dev.079087 (2013).

15 Zellag, R. M. et al. CentTracker: a trainable, machine-learning-based tool for large-scale analyses of Caenorhabditis elegans germline stem cell mitosis. Mol Biol Cell 32, 915930, doi:10.1091/mbc.E20-11-0716 (2021).

16 Henderson, S. T., Gao, D., Lambie, E. J. \& Kimble, J. lag-2 may encode a signaling ligand for the GLP-1 and LIN-12 receptors of C. elegans. Development 120, 2913-2924 (1994).

17 Austin, J. \& Kimble, J. glp-1 is required in the germ line for regulation of the decision between mitosis and meiosis in C. elegans. Cell 51, 589-599, doi:10.1016/00928674(87)90128-0 (1987).

18 Chen, J. et al. GLP-1 Notch-LAG-1 CSL control of the germline stem cell fate is mediated by transcriptional targets lst-1 and sygl-1. PLoS Genet 16, e1008650, doi:10.1371/journal.pgen.1008650 (2020). 
19 Lee, C., Sorensen, E. B., Lynch, T. R. \& Kimble, J. C. elegans GLP-1/Notch activates transcription in a probability gradient across the germline stem cell pool. Elife $\mathbf{5}$, doi:10.7554/eLife.18370 (2016).

20 Shin, H. et al. SYGL-1 and LST-1 link niche signaling to PUF RNA repression for stem cell maintenance in Caenorhabditis elegans. PLoS Genet 13, e1007121, doi:10.1371/journal.pgen.1007121 (2017).

21 Haupt, K. A. et al. The molecular basis of LST-1 self-renewal activity and its control of stem cell pool size. Development 146, doi:10.1242/dev.181644 (2019).

22 Hubbard, E. J. A. \& Schedl, T. Biology of the Caenorhabditis elegans Germline Stem Cell System. Genetics 213, 1145-1188, doi:10.1534/genetics.119.300238 (2019).

23 Yamashita, Y. M., Fuller, M. T. \& Jones, D. L. Signaling in stem cell niches: lessons from the Drosophila germline. J Cell Sci 118, 665-672, doi:10.1242/jcs.01680 (2005).

24 Drummond-Barbosa, D. \& Spradling, A. C. Stem cells and their progeny respond to nutritional changes during Drosophila oogenesis. Dev Biol 231, 265-278, doi:10.1006/dbio.2000.0135 (2001).

25 LaFever, L. \& Drummond-Barbosa, D. Direct control of germline stem cell division and cyst growth by neural insulin in Drosophila. Science 309, 1071-1073, doi:10.1126/science.1111410 (2005).

26 Murphy, C. T. \& Hu, P. J. in Wormbook (ed ed. The C. elegans Research Community) (WormBook, doi/10.1895/wormbook.1.164.1, 2013).

27 Michaelson, D., Korta, D. Z., Capua, Y. \& Hubbard, E. J. Insulin signaling promotes germline proliferation in C. elegans. Development 137, 671-680, doi:10.1242/dev.042523 (2010).

28 Narbonne, P., Maddox, P. S. \& Labbe, J. C. DAF-18/PTEN locally antagonizes insulin signalling to couple germline stem cell proliferation to oocyte needs in C. elegans. Development 142, 4230-4241, doi:10.1242/dev.130252 (2015).

29 Narbonne, P., Maddox, P. S. \& Labbe, J. C. DAF-18/PTEN signals through AAK1/AMPK to inhibit MPK-1/MAPK in feedback control of germline stem cell proliferation. PLoS Genet 13, e1006738, doi:10.1371/journal.pgen.1006738 (2017).

30 Fukuyama, M., Rougvie, A. E. \& Rothman, J. H. C. elegans DAF-18/PTEN mediates nutrient-dependent arrest of cell cycle and growth in the germline. Curr Biol 16, 773-779, doi:10.1016/j.cub.2006.02.073 (2006).

31 Fukuyama, M. et al. C. elegans AMPKs promote survival and arrest germline development during nutrient stress. Biol Open 1, 929-936, doi:10.1242/bio.2012836 (2012).

32 Narbonne, P., Hyenne, V., Li, S., Labbe, J. C. \& Roy, R. Differential requirements for STRAD in LKB1-dependent functions in C. elegans. Development 137, 661-670, doi:10.1242/dev.042044 (2010).

33 Narbonne, P. \& Roy, R. Inhibition of germline proliferation during C. elegans dauer development requires PTEN, LKB1 and AMPK signalling. Development 133, 611-619, doi:10.1242/dev.02232 (2006).

34 Tenen, C. C. \& Greenwald, I. Cell Non-autonomous Function of daf-18/PTEN in the Somatic Gonad Coordinates Somatic Gonad and Germline Development in C. elegans Dauer Larvae. Curr Biol 29, 1064-1072 e1068, doi:10.1016/j.cub.2019.01.076 (2019).

35 Gan, B. et al. Lkb1 regulates quiescence and metabolic homeostasis of haematopoietic stem cells. Nature 468, 701-704, doi:10.1038/nature09595 (2010). 
36 Gurumurthy, S. et al. The Lkb1 metabolic sensor maintains haematopoietic stem cell survival. Nature 468, 659-663, doi:10.1038/nature09572 (2010).

37 Nakada, D., Saunders, T. L. \& Morrison, S. J. Lkb1 regulates cell cycle and energy metabolism in haematopoietic stem cells. Nature 468, 653-658, doi:10.1038/nature09571 (2010).

38 Yilmaz, O. H. et al. Pten dependence distinguishes haematopoietic stem cells from leukaemia-initiating cells. Nature 441, 475-482, doi:10.1038/nature04703 (2006).

39 Zhang, J. et al. PTEN maintains haematopoietic stem cells and acts in lineage choice and leukaemia prevention. Nature 441, 518-522, doi:10.1038/nature04747 (2006).

40 Tomasetti, C. \& Vogelstein, B. Cancer etiology. Variation in cancer risk among tissues can be explained by the number of stem cell divisions. Science 347, 78-81, doi:10.1126/science.1260825 (2015).

41 Morgan, D. E., Crittenden, S. L. \& Kimble, J. The C. elegans adult male germline: stem cells and sexual dimorphism. Dev Biol 346, 204-214, doi:10.1016/j.ydbio.2010.07.022 (2010).

42 Cinquin, A. et al. Intermittent Stem Cell Cycling Balances Self-Renewal and Senescence of the C. elegans Germ Line. PLoS Genet 12, e1005985, doi:10.1371/journal.pgen.1005985 (2016).

43 Conboy, I. M. et al. Rejuvenation of aged progenitor cells by exposure to a young systemic environment. Nature 433, 760-764, doi:10.1038/nature03260 (2005).

44 Haase, V. H. Hypoxic regulation of erythropoiesis and iron metabolism. Am J Physiol Renal Physiol 299, F1-13, doi:10.1152/ajprenal.00174.2010 (2010).

45 Jiang, H. et al. Cytokine/Jak/Stat signaling mediates regeneration and homeostasis in the Drosophila midgut. Cell 137, 1343-1355, doi:10.1016/j.cell.2009.05.014 (2009).

46 Robinson-Thiewes, S. et al. Non-autonomous regulation of germline stem cell proliferation by somatic MPK-1/MAPK activity in C. elegans. Cell Reports 35 (2021).

47 Murray, P. \& Edgar, D. The regulation of embryonic stem cell differentiation by leukaemia inhibitory factor (LIF). Differentiation 68, 227-234, doi:10.1046/j.14320436.2001.680410.x (2001).

48 Matsuda, T. et al. STAT3 activation is sufficient to maintain an undifferentiated state of mouse embryonic stem cells. EMBO J 18, 4261-4269, doi:10.1093/emboj/18.15.4261 (1999).

49 Ying, Q. L., Nichols, J., Chambers, I. \& Smith, A. BMP induction of Id proteins suppresses differentiation and sustains embryonic stem cell self-renewal in collaboration with STAT3. Cell 115, 281-292, doi:10.1016/s0092-8674(03)00847-x (2003).

50 Ying, Q. L. et al. The ground state of embryonic stem cell self-renewal. Nature 453, 519523, doi:10.1038/nature06968 (2008).

51 Byrd, D. T., Knobel, K., Affeldt, K., Crittenden, S. L. \& Kimble, J. A DTC niche plexus surrounds the germline stem cell pool in Caenorhabditis elegans. PLoS One 9, e88372, doi:10.1371/journal.pone.0088372 (2014).

52 Berry, L. W., Westlund, B. \& Schedl, T. Germ-line tumor formation caused by activation of glp-1, a Caenorhabditis elegans member of the Notch family of receptors. Development 124, 925-936 (1997).

53 Kostic, I., Li, S. \& Roy, R. cki-1 links cell division and cell fate acquisition in the C. elegans somatic gonad. Dev Biol 263, 242-252, doi:10.1016/j.ydbio.2003.07.001 (2003). 
54 Pepper, A. S., Killian, D. J. \& Hubbard, E. J. Genetic analysis of Caenorhabditis elegans glp-1 mutants suggests receptor interaction or competition. Genetics 163, 115-132 (2003).

55 Francis, R., Barton, M. K., Kimble, J. \& Schedl, T. gld-1, a tumor suppressor gene required for oocyte development in Caenorhabditis elegans. Genetics 139, 579-606 (1995).

56 Francis, R., Maine, E. \& Schedl, T. Analysis of the multiple roles of gld-1 in germline development: interactions with the sex determination cascade and the glp-1 signaling pathway. Genetics 139, 607-630 (1995).

57 Voog, J. \& Jones, D. L. Stem cells and the niche: a dynamic duo. Cell Stem Cell 6, 103115, doi:10.1016/j.stem.2010.01.011 (2010).

58 Yan, D. et al. A regulatory network of Drosophila germline stem cell self-renewal. Dev Cell 28, 459-473, doi:10.1016/j.devcel.2014.01.020 (2014).

59 Kiger, A. A., Jones, D. L., Schulz, C., Rogers, M. B. \& Fuller, M. T. Stem cell selfrenewal specified by JAK-STAT activation in response to a support cell cue. Science 294, 2542-2545, doi:10.1126/science.1066707 (2001).

60 Parrott, B. B., Hudson, A., Brady, R. \& Schulz, C. Control of germline stem cell division frequency--a novel, developmentally regulated role for epidermal growth factor signaling. PLoS One 7, e36460, doi:10.1371/journal.pone.0036460 (2012).

61 Griffiths A. J. F., Miller J. H., Suzuki D. T. \& al. in An introduction to genetic analysis, 7th Edition (ed W. H. Freeman) (2000).

62 Crane, G. M., Jeffery, E. \& Morrison, S. J. Adult haematopoietic stem cell niches. Nat Rev Immunol 17, 573-590, doi:10.1038/nri.2017.53 (2017).

63 Crittenden, S. L. et al. A conserved RNA-binding protein controls germline stem cells in Caenorhabditis elegans. Nature 417, 660-663, doi:10.1038/nature754 (2002).

64 Byerly, L., Cassada, R. C. \& Russell, R. L. The life cycle of the nematode Caenorhabditis elegans. I. Wild-type growth and reproduction. Dev Biol 51, 23-33, doi:10.1016/00121606(76)90119-6 (1976).

65 McCarter, J., Bartlett, B., Dang, T. \& Schedl, T. On the control of oocyte meiotic maturation and ovulation in Caenorhabditis elegans. Dev Biol 205, 111-128, doi:10.1006/dbio.1998.9109 (1999).

66 Riddle, D., Blumenthal, T., Meyer, B. \& al., e. in C. elegans II. 2nd edition. (ed Cold Spring Harbor Laboratory Press.) Ch. Section IX, Oocyte Development, Maturation, and Ovulation., (1997).

67 Fox, P. M. et al. Cyclin E and CDK-2 regulate proliferative cell fate and cell cycle progression in the C. elegans germline. Development 138, 2223-2234, doi:10.1242/dev.059535 (2011).

68 Gumienny, T. L., Lambie, E., Hartwieg, E., Horvitz, H. R. \& Hengartner, M. O. Genetic control of programmed cell death in the Caenorhabditis elegans hermaphrodite germline. Development 126, 1011-1022 (1999).

69 Wolke, U., Jezuit, E. A. \& Priess, J. R. Actin-dependent cytoplasmic streaming in C. elegans oogenesis. Development 134, 2227-2236, doi:10.1242/dev.004952 (2007).

70 Angelo, G. \& Van Gilst, M. R. Starvation protects germline stem cells and extends reproductive longevity in C. elegans. Science 326, 954-958, doi:10.1126/science.1178343 (2009). 
71 Schedl, T. \& Kimble, J. fog-2, a germ-line-specific sex determination gene required for hermaphrodite spermatogenesis in Caenorhabditis elegans. Genetics 119, 43-61 (1988).

72 Miller, M. A. et al. A sperm cytoskeletal protein that signals oocyte meiotic maturation and ovulation. Science 291, 2144-2147, doi:10.1126/science.1057586 (2001).

73 Kim, S., Spike, C. \& Greenstein, D. Control of oocyte growth and meiotic maturation in Caenorhabditis elegans. Adv Exp Med Biol 757, 277-320, doi:10.1007/978-1-4614-40154_10 (2013).

74 Detwiler, M. R., Reuben, M., Li, X., Rogers, E. \& Lin, R. Two zinc finger proteins, OMA-1 and OMA-2, are redundantly required for oocyte maturation in C. elegans. Dev Cell 1, 187-199 (2001).

75 van der Flier, L. G. \& Clevers, H. Stem cells, self-renewal, and differentiation in the intestinal epithelium. Annu Rev Physiol 71, 241-260, doi:10.1146/annurev.physiol.010908.163145 (2009).

76 Barker, N. et al. Identification of stem cells in small intestine and colon by marker gene Lgr5. Nature 449, 1003-1007, doi:10.1038/nature06196 (2007).

77 Gajendran, M., Loganathan, P., Catinella, A. P. \& Hashash, J. G. A comprehensive review and update on Crohn's disease. Dis Mon 64, 20-57, doi:10.1016/j.disamonth.2017.07.001 (2018).

78 Yilmaz, B. \& Li, H. Gut Microbiota and Iron: The Crucial Actors in Health and Disease. Pharmaceuticals (Basel) 11, doi:10.3390/ph11040098 (2018).

79 Saunders, D. R., Haggitt, R. C., Kimmey, M. B. \& Silverstein, F. E. Morphological consequences of bisacodyl on normal human rectal mucosa: effect of a prostaglandin E1 analog on mucosal injury. Gastrointest Endosc 36, 101-104, doi:10.1016/s00165107(90)70960-1 (1990).

80 Zhang, P. et al. An SH3PX1-Dependent Endocytosis-Autophagy Network Restrains Intestinal Stem Cell Proliferation by Counteracting EGFR-ERK Signaling. Dev Cell 49, 574-589 e575, doi:10.1016/j.devcel.2019.03.029 (2019).

81 Barker, N. et al. Crypt stem cells as the cells-of-origin of intestinal cancer. Nature 457, 608-611, doi:10.1038/nature07602 (2009).

82 Leite-Gomes, E. et al. Bringing to Light the Risk of Colorectal Cancer in Inflammatory Bowel Disease: Mucosal Glycosylation as a Key Player. Inflamm Bowel Dis, doi:10.1093/ibd/izab291 (2021).

83 Croft, D. P., Brent, L. J., Franks, D. W. \& Cant, M. A. The evolution of prolonged life after reproduction. Trends Ecol Evol 30, 407-416, doi:10.1016/j.tree.2015.04.011 (2015).

84 Tomasetti, C. et al. Role of stem-cell divisions in cancer risk. Nature 548, E13-E14, doi:10.1038/nature23302 (2017).

85 Tomasetti, C., Li, L. \& Vogelstein, B. Stem cell divisions, somatic mutations, cancer etiology, and cancer prevention. Science 355, 1330-1334, doi:10.1126/science.aaf9011 (2017).

86 Bajaj, J., Diaz, E. \& Reya, T. Stem cells in cancer initiation and progression. $J$ Cell Biol 219, doi:10.1083/jcb.201911053 (2020).

87 Tan, B. T., Park, C. Y., Ailles, L. E. \& Weissman, I. L. The cancer stem cell hypothesis: a work in progress. Lab Invest 86, 1203-1207, doi:10.1038/labinvest.3700488 (2006).

88 Hemminki, A. et al. A serine/threonine kinase gene defective in Peutz-Jeghers syndrome. Nature 391, 184-187, doi:10.1038/34432 (1998). 
89 Jenne, D. E. et al. Peutz-Jeghers syndrome is caused by mutations in a novel serine threonine kinase. Nat Genet 18, 38-43, doi:10.1038/ng0198-38 (1998).

90 Wirtzfeld, D. A., Petrelli, N. J. \& Rodriguez-Bigas, M. A. Hamartomatous polyposis syndromes: molecular genetics, neoplastic risk, and surveillance recommendations. Ann Surg Oncol 8, 319-327, doi:10.1007/s10434-001-0319-7 (2001).

91 Blaj, C. et al. Oncogenic Effects of High MAPK Activity in Colorectal Cancer Mark Progenitor Cells and Persist Irrespective of RAS Mutations. Cancer Res 77, 1763-1774, doi:10.1158/0008-5472.CAN-16-2821 (2017).

92 Mahoney, C. L. et al. LKB1/KRAS mutant lung cancers constitute a genetic subset of NSCLC with increased sensitivity to MAPK and mTOR signalling inhibition. $\mathrm{Br} J$ Cancer 100, 370-375, doi:10.1038/sj.bjc.6604886 (2009).

93 Mathew, G. et al. Targeting of Ras-mediated FGF signaling suppresses Pten-deficient skin tumor. Proc Natl Acad Sci U S A 113, 13156-13161, doi:10.1073/pnas.1604450113 (2016).

94 Hansen, D., Hubbard, E. J. \& Schedl, T. Multi-pathway control of the proliferation versus meiotic development decision in the Caenorhabditis elegans germline. Dev Biol 268, 342357, doi:10.1016/j.ydbio.2003.12.023 (2004). 